TRANSACTIONS OF THE

AMERICAN MATHEMATICAL SOCIETY

Volume 186, December 1973

\title{
THE VALUES OF EXPONENTIAL POLYNOMIALS AT ALGEBRAIC POINTS. I
}

BY

\section{CARLOS JULIO MORENO}

ABSTRACT. A strengthening of Siegel's proof of the Hermite-Lindemann Theorem is given. The results are used to obtain lower bounds for the values of exponential polynomials at algebraic points. The question of how well the root of an exponential polynomial can be approximated by algebraic numbers is considered, and a lower bound is obtained for the absolute value of the difference between a root of the exponential polynomial and an algebraic number.

1. Introduction. In this article we investigate the values at algebraic points of exponential polynomials of the type

$$
\phi(z)=\sum_{k=1}^{m} A_{k}(z) \exp \left\{\alpha_{k} z\right\}
$$

where the frequencies $a_{k}(1 \leq k \leq m)$ are algebraic numbers, and the $A_{k}(z)$ $(1 \leq k \leq m)$ are polynomials in $z$ whose coefficients are also algebraic numbers. The results which we prove are generalizations of the Hermite-Lindemann Theorem to the effect that the values taken by the exponential function at distinct algebraic points are linearly independent over the field of algebraic numbers. The central result of this article (see $\$ 2$, Theorem 1 ) says that given an algebraic number $\beta$ of height $H(\beta)$, an explicit lower bound for $|\phi(\beta)|$ can be obtained as a function of $H(\beta)$. The method of proof which we use to establish this result is a generalization of a technique that Siegel had invented to deal with exponential polynomials of the type

$$
\phi^{*}(z)=\sum_{k=1}^{m} A_{k}(z) \exp \left\{\alpha_{k}\right\},
$$

where the $a_{k}$ and $A_{k}(z)$ are defined as above. The method of Siegel was subsequently improved by Mahler who was in fact able to show that given an algebraic number $\beta$ of height $H(\beta)$, a constant $\Lambda$ can be found which depends only on the exponential polynomial $\phi^{*}(z)$ and the degree of $\beta$ such that

Received by the editors December 7, 1971.

AMS (MOS) subject classifications (1970). Primary 10Fxx; Secondary 10F35. '

Key words and phrases. Algebraic, diophantine, approximation, exponential polynomial, Hermite-Lindemann Theorem. 


$$
\left|\phi^{*}(\beta)\right| \geq(H(\beta)+1)^{-\Lambda} \text {. }
$$

A simple application of Taylor's Theorem leads to estimates of how well a root of the exponential polynomial $\phi^{*}(z)$ can be approximated by algebraic numbers.

The methods developed in this paper can also be used to investigate the values taken by polynomials of the form

$$
\phi_{E}(z)=\sum_{k=1}^{m} A_{k}(z) E_{k}(z)
$$

where the $A_{k}(z)$ are defined as above and the $E_{k}(z)$ are the $E$-functions of Siegel. In a subsequent paper we plan to investigate this and related problems by using results of Šidlovskii [9] and Lang [4] concerning the measure of transcendency of $E$-functions. Again these results lead to estimates of how well a root of an $E$-polynomial $\phi_{E}(z)$ can be approximated by algebraic numbers.

2. Statement of results. The following is the main result of this paper.

Theorem 1. Let $\phi(z)$ be the exponential polynomial

$$
\phi(z)=\sum_{k=1}^{m} A_{k}(z) \exp \left\{\alpha_{k} z\right\},
$$

where $a_{k}, A_{k}(z)(1 \leq k \leq m)$ are respectively algebraic numbers and polynomials in $z$ with algebraic coefficients. If $\beta$ is an algebraic number of beight $H=H(\beta)$, then

$$
|\phi(\beta)| \geq \exp \left\{-\Lambda H^{7}\right\}
$$

where $\Lambda$ is a constant depending only on the polynomial $\phi(z)$ and the degree of $\beta$.

The following result is an easy consequence of Theorem 1 .

Theorem 2. If $\beta$ is an algebraic number of beight $H=H(\beta)$ and $z$ is a root of $\phi(z)=0$, then

$$
|z-\beta| \geq \exp \left\{-\Lambda_{0} H^{7}\right\}
$$

where $\Lambda_{0}$ is a constant depending only on the polynomial $\phi(z)$ and the degree of $\beta$.

Theorem 2 improves on the results of Fel'dman [1] and Mardohai-Boltouski [7].

3. Preliminary results. The main idea of the proof of Theorem 1 is a combination of ideas in Siegel ([10], [11]) and Mahler [6]. Several presentations of 
Siegel's method have been given in the literature, including a simplified version due to Mahler. Our treatment is modeled on that of Gel' fond [2, Chapter II, \$2].

Without loss of generality we may assume that the coefficients of the polynomials $A_{k}(z)$ are algebraic integers of a number field $K$ of degree $\nu$, which we will hold fixed throughout our discussion. We suppose further that $a_{k} \in K$ $(1 \leq k \leq m)$. Let $w_{1}, \cdots, w_{\nu}$ be a basis for the field $K$ consisting entirely of algebraic integers, i.e., an integral basis, and write the polynomial $\phi(z)$ as

$$
\phi(z)=\sum_{k_{1}=0}^{\sigma} \cdots \sum_{k_{\nu}=0}^{\sigma} A_{k_{1}}, \cdots, k_{\nu}(z) \exp \left\{\left(k_{1} w_{1}+\cdots+k_{\nu} w_{\nu}\right) z / \delta\right\}
$$

where the polynomials $A_{k_{1}, \ldots, k \nu}(z)$ are those appearing in (1) suitably indexed, $\delta$ is a rational integer and $\sigma$ is a positive integer which depends only on $a_{1}, \ldots$, $a_{m}$ and the choice of basis elements $w_{1}, \ldots, w_{\nu}$. We assume that for each poly. nomial coefficient in (1), $A_{k}(z)=\sum_{j=1}^{d} a_{k, j} z^{j}$, we have

$$
\left|a_{k, j}\right| \leq \max _{1 \leq j \leq d ; 1 \leq k \leq m}\left|\overline{a_{k, j}}\right|=A,
$$

where the symbol $|\bar{\alpha}|$ means the largest of the absolute values of the conjugates of $\alpha$ and

$$
\left.d=\operatorname{Max} \text { (degree } A_{1}(z), \ldots \text {, degree } A_{m}(z) \text {; degree } \beta\right) \text {. }
$$

In the proof of Theorem 1 we may assume that the $a_{k}(1 \leq k \leq m)$ are algebraic integers in $K$, that is to say, we may assume that in (2) $\delta=1$. In fact, if $\delta \neq 1$, we may prove Theorem 1 for the polynomial $\phi_{\delta}(z)=\phi(\delta z)$ and then consider the value of $\phi_{\delta}(z)$ at the algebraic point $\beta / \delta$.

We will need the following result.

Lemma. Let the coefficients $a_{k, s}$ of the linear forms

$$
L_{k}=a_{k, 1} x_{1}+\cdots+a_{k, q^{x}} \quad(1 \leq k \leq p)
$$

be in the algebraic number field $K$ of degree $\nu$. Suppose that $p<q$ and $\left|A_{k, s}\right|$ $\leq A(1 \leq k \leq p, 1 \leq s \leq q)$. Then there is a nonzero solution $\left(x_{1}, \ldots, x_{q}\right)$ of the system of equations $L_{k}=0(1 \leq k \leq p)$ in integers of the field $K$ satisfying

$$
\overline{\left|x_{k}\right|} \leq c_{1}\left(c_{2} q A\right)^{p /(q-p)}+c_{1},
$$

where $c_{1}$ and $c_{2}$ are constants depending only on $K$.

The proof of this Lemma is a straightforward application of the pigeon-hole principle and can be found for example in Lang [4, p. 4].

4. Proof of Theorem 1. The proof will be by contradiction. We assume that for some constant $c$, whose value will be determined at the end of the argument, 
and no matter how large we take $\Lambda$, we can always find an algebraic number $\beta$ of large height such that

$$
\phi(\beta) \ll \exp \left\{-\lambda H^{c}\right\} .
$$

In the spirit of Gel'fond [2, Chapter II, \$2] we shall subdivide the proof of the impossibility of (4) into six steps.

Step One. We enumerate the $q=(p+1)^{\nu}$ numbers

$$
k_{1} w_{1}+\cdots+k_{\nu} w_{\nu}, \quad 0 \leq k_{i} \leq p, \quad 1 \leq i \leq \nu,
$$

where $p$ is a sufficiently large positive integer, in any order to form a sequence $\lambda_{1}=0, \lambda_{2}, \cdots, \lambda_{q}$ with the first $\xi=(\sigma+1)^{\nu}$ of them coinciding with the set of numbers $k_{1} w_{1}+\cdots+k_{\nu} w_{\nu}, 0 \leq k_{i} \leq \sigma, 1 \leq i \leq \nu$.

Thus we may write the polynomial

$$
\phi(z)=\sum_{k_{1}=0}^{\sigma} \cdots \sum_{k_{\nu}=0}^{\sigma} A_{k_{1}, \cdots, k_{\nu}}(z) \exp \left\{\left(k_{1} w_{1}+\cdots+k_{\nu} w_{\nu}\right) z\right\}
$$

as

$$
\phi_{1}(z)=\sum_{k=1}^{q} A_{1, k}(s) \exp \left\{\lambda_{k} z\right\}, \quad A_{1, k}(z)=0 \text { for } k>\xi
$$

If we multiply the relation (4) by the numbers

$$
\exp \left\{\left(k_{1} w_{1}+\cdots+k_{\nu} w_{\nu}\right) \beta\right\}, \quad 0 \leq k_{i} \leq p-\sigma, 1 \leq i \leq \nu,
$$

we shall obtain a new set of inequalities of the type

$$
\phi_{j}(\beta)=\sum_{k=1}^{q} A_{j, k}(\beta) \exp \left\{\lambda_{k} \beta\right\} \leq \exp \left\{-\Lambda H^{c}\right\},
$$

with $1 \leq j \leq(p+1-\sigma)^{\nu}=\tau$. Clearly, the constant $\Lambda$ in (5) now depends on $p$. This will do no harm to the argument, since $p$ will be a parameter to be chosen later as a function of $H$ which will not grow faster than some power of $\log H$.

We now claim that the matrix $\left(A_{j . k}(\beta)\right)(1 \leq j \leq \tau, 1 \leq k \leq q)$, formed with the coefficients of the linear forms $\phi_{j}(\beta)$ in (5) has rank $\tau$. This follows easily from the fact that the $w_{i}(1 \leq i \leq \nu)$ are linearly independent which implies that the matrix $\left(A_{j, k}(\beta)\right)$ is semidiagonal after a suitable interchange of rows. The next step consists in producing a new set of $q$ independent linear forms in the exponentials $\exp \left\{\lambda_{k} \beta\right\}(1 \leq k \leq q)$, from which a set of $q-r$ forms can be selected so as to complete the system in (5).

Step Two. The following is Siegel's method for constructing $q$ independent linear forms in the exponentials $\exp \left\{\lambda_{k} \beta\right\}(1 \leq k \leq q)$ as outlined in Gel'fond 
[2, Chapter II, \$2]. The idea is to construct a function $f(z)$ which is a linear combination of the exponentials $\exp \left\{\lambda_{k} z\right\}(1 \leq k \leq q)$ and polynomials $P_{k}(z)$, each of degree at most $N$ and such that

$$
f(z)=\sum_{k=1}^{q} P_{k}(z) \exp \left\{\lambda_{k} z\right\}
$$

has a zero at the origin of high multiplicity.

Remark. Hopefully a refinement of this construction may lead to an improvement of the final estimate. In fact, one can construct lots of linear forms by requiring that the function $f(z)$ vanish not only at the origin but at other points located symmetrically about the origin. For example, we could take the Gaussian integers $\rho=m+n i, m^{2}+n^{2} \leq Q$, and require that the function

$$
f(z)=\sum_{m=1}^{q} P_{m}(z) \exp \left\{\lambda_{m} z\right\}
$$

vanish to a high order at all points $\rho$. Actually one only needs that the numbers $f\left(\rho_{1}\right), f\left(\rho_{2}\right), \ldots$, and some higher order derivatives be small. In carrying out this plan there is one difficulty which we have not been able to overcome and that is that the point $z=0$ is the only algebraic point of the function $\left(z, e^{z}\right)$. We hope to investigate this problem in a subsequent paper.

Let $N$ be a positive integer which will be chosen later to be large compared to $q$. Form the function

$$
f(z)=\sum_{m=1}^{q} P_{m}(z) \exp \left\{\lambda_{m} z\right\}
$$

where

$$
P_{m}(z)=N ! \sum_{k=0}^{N} c_{m, k} \frac{z^{k}}{k !} .
$$

If $f(z)$ is to have a zero of high order at the origin, we must be able to select the coefficients $c_{m, k}$ suitably so that the power series expansion of $f(z)$ about the origin begins with a high power of $z$. By expanding the exponentials $\exp \left\{\lambda_{m} z\right\}$ in powers of $z$ we can write $f(z)$ as

$$
\begin{aligned}
f(z) & =\sum_{m=1}^{q}\left(N ! \sum_{k=0}^{N} c_{m, k} \frac{z^{k}}{k !} \sum_{\nu=0}^{\infty} \frac{\lambda_{m}^{v} z^{\nu}}{v !}\right) \\
& =\sum_{m=1}^{q} \sum_{w=0}^{\infty}\left(\sum_{0 \leq k \leq N ; 0 \leq v \leq w ; k+v=w} c_{m, k} \lambda_{m}^{v} \frac{N ! w !}{k ! v !}\right) \frac{z^{w}}{w !}
\end{aligned}
$$

if we now interchange the order of summation we get 
(8)

$$
\begin{aligned}
& =\sum_{w=0}^{\infty}\left(\sum_{m=1}^{q} \sum_{v=0}^{w} c_{m, w-v} \lambda_{m}^{v} \frac{N ! w !}{(w-v) ! v !}\right) \frac{z^{w}}{w !} \\
& =N ! \sum_{w=0}^{\infty}\left(\sum_{m=1}^{q} \sum_{v=0}^{w} c_{m, w-v} \lambda^{v}\left(\begin{array}{l}
w \\
v
\end{array}\right)\right) \frac{z^{w}}{w !}
\end{aligned}
$$

where $c_{m, w-v}=0$ for $w-v>N$ and the symbol $\left(\begin{array}{c}w \\ v\end{array}\right)$ is the binomial coefficient. To construct the polynomials $P_{m}(z)(1 \leq m \leq q)$, each of degree $N$, we think of the coefficients $c_{m, k}$ as unknowns and observe that if $f(z)$ is to have a zero at $z=0$ of multiplicity $M$, then the following system of linear equations in the $c_{m, k}$ must hold:

$$
\sum_{m=1}^{q} \sum_{v=0}^{w} \lambda_{m}^{v}\left(\begin{array}{l}
w \\
v
\end{array}\right) c_{m, w-v}=0, \quad 0 \leq w \leq M-1 .
$$

To apply Lemma 1, we must first find bounds for the coefficients in (9). Clearly we have $(\underset{v}{w}) \leq 2^{w} \leq 2^{M}(0 \leq w \leq M-1)$ and $\left|\lambda_{m}\right| \leq c_{3} p(1 \leq m \leq q)$. Therefore we have

$$
A=\operatorname{Max}_{w, m, v}\left(\begin{array}{l}
w \\
v
\end{array}\right) \overline{\left|\lambda_{m}^{v}\right|} \leq c_{4}\left(c_{s} p\right)^{M} .
$$

Now, the total number of equations in (9) is $M$ and the number of unknowns is $(N+1) q$, hence the Lemma guarantees the existence of a nonzero solution to the system in (9) in integers $c_{m, k}$ of the field $K$ and satisfying the inequalities

$$
\left|\overline{c_{m, k}}\right| \leq c_{6}\left(c_{7} N q\left(c_{5} p\right)^{M}\right)^{M /((N+1) q-M)}
$$

provided that $M<(N+1) q$.

We have thus constructed a function $f(z)$ of the form

$$
f(z)=z^{M} \psi(z)
$$

where $\psi(z)$ is an entire function.

To complete the second step we now obtain bounds for the value of the function $\psi(z)$ at points near the origin. From (8) we see that $f(z)$ can be written as

$$
f(z)=z^{M} \sum_{b=0}^{\infty}\left(\sum_{m=1}^{q} \sum_{v=0}^{M+b} N ! c_{m, M+b-v} \lambda_{m}^{v}\left(\begin{array}{c}
M+b \\
v
\end{array}\right)\right) \frac{z^{b}}{(M+b) !},
$$

and thus

$$
\psi(z)=N ! \sum_{b=0}^{\infty}\left(\sum_{m=1}^{q} \sum_{v=0}^{M+b} c_{m, M+b-v} \lambda_{m}^{v}\left(\begin{array}{c}
M+b \\
v
\end{array}\right)\right) \frac{z^{b}}{(M+b) !} .
$$

Taking absolute values and putting $B=\operatorname{Max}_{k, m}\left|\overline{c_{m, k}}\right|$, we obtain

$$
|\psi(z)| \leq \frac{N !}{M !} \sum_{b=0}^{\infty} q(M+b) 2^{M+h}\left(c_{3} p\right)^{M+b} B \frac{|z|^{b} M !}{(M+b) !} .
$$


We now use the trivial estimates $(M+b) 2^{M+b}<3^{M+b}$ and $M ! b ! /(M+b) ! \leq 1$ in (12) to get

$$
\begin{aligned}
|\psi(z)| & \leq \frac{N !}{M !} B q\left(c_{8} p\right)^{M} \sum_{b=0}^{\infty} \frac{\left(c_{8} p|z|\right)^{b}}{b !} \\
& \leq \frac{N !}{M !} B q\left(c_{8} p\right)^{M} \exp \left\{c_{8} p|z|\right\} .
\end{aligned}
$$

The size of the constant $c_{8}$ is of some importance if one wants to obtain an estimate like that in Theorem 1 with an explicit dependence on the degree of $\beta$. A more careful analysis of the above estimates gives that $c_{8}=3 \nu \omega$, where $\nu$ is the degree of $K$ over the rationals and $\omega$ is an upper bound for the absolute values of all the conjugates of the basis $w_{1}, \ldots, w_{\nu}$ in $K$.

Later on it will be advantageous if the exponent in (10) is of the order of magnitude of $q$, i.e., $M /((N+1) q-M)=c_{9} q$, that is to say $M$ should be of the order of magnitude of $N q$. This we accomplish by taking $M=N(q-1)+2 q$. The above choice of $M$ gives the following bound for $B$ in (13), or equivalently for $\left|\overline{c_{m, k}}\right|$ in (10), $B \leq c_{6} \exp \left\{4 N q^{2} \log p\right\}$, provided that $p \geq c_{9}$ where $c_{9}$ is an effectively computable constant. Finally we get

$$
|\psi(z)| \leq \exp \left\{-q(N / 2) \log N+c_{8} p|z|+4 N q^{2} \log p\right\}
$$

provided $p \geq c_{10}$.

Step Three. We now differentiate the function $f(z)$ to obtain other linear forms in the exponentials $\exp \left\{\lambda_{m} z\right\} \quad(1 \leq m \leq q)$. Put

$$
L_{s}(z)=f^{(s)}(z)=\sum_{m=1}^{q} U_{s, m}(z) \exp \left\{\lambda_{m} z\right\}, \quad s=0,1, \ldots, N-1,
$$

where

$$
U_{s, m}(z)=\sum_{k=0}^{N} c_{m, s, k} z^{k} .
$$

To find upper bounds for the coefficients $c_{m, s, k}$ we put $D=d / d z$ and use Leibniz' rule to obtain

$$
\begin{aligned}
L_{s}(z) & =D^{s} f(z)=\sum_{m=1}^{q} D^{s}\left(P_{m}(z) \exp \left\{\lambda_{m} z\right\}\right) \\
& =\sum_{m=1}^{q}\left(\sum_{r=0}^{s}\left(\begin{array}{l}
s \\
r
\end{array}\right) D^{r} P_{m}(z) \lambda_{m}^{s-r}\right) \exp \left\{\lambda_{m} z\right\} \\
& =\sum_{m=1}^{q}\left(\sum_{r=0}^{s}\left(\begin{array}{l}
s \\
r
\end{array}\right) \lambda_{m}^{s-r} D^{r} \sum_{k=0}^{N} N ! c_{m, k} \frac{z^{k}}{k !}\right) \exp \left\{\lambda_{m} z\right\}
\end{aligned}
$$


The coefficients in each of the polynomials

$$
N ! \sum_{k=0}^{N} c_{m, k} D^{r} \frac{z^{k}}{k !}, \quad r \leq s,
$$

are certainly bounded by $N ! \Sigma_{k=0}^{N}\left|\overline{c_{m, k}}\right|$; if we estimate the other terms in (16) trivially we obtain that the coefficients in the polynomials in (15) are bounded by provided $p>c_{11}$.

$$
\overline{\left|c_{m, s, k}\right|} \leq \exp \left\{2 N \log N+4 N q^{2} \log p\right\}
$$

We would now like to prove that given an arbitrary point $z \neq 0$, among the $N$ forms $L_{0}(z), L_{1}(z), \ldots, L_{N-1}(z), q$ of these are linearly independent, that is to say, the matrix of coefficients $\left(U_{s, m}(z)\right)$ is nonsingular.

We first establish that none of the polynomials $P_{m}(z)$ in

$$
f(z)=\sum_{m=1}^{q} P_{m}(z) \exp \left\{\lambda_{m} z\right\}
$$

can vanish identically. Recall that $f(z)$ has a zero at $z=0$ of multiplicity $M$, and suppose that on the contrary

$$
P_{1}(z) \equiv P_{2}(z) \equiv \cdots \equiv P_{\eta}(z) \equiv 0
$$

are the only polynomials that vanish identically. To both sides of the equality

$$
\psi(z) z^{M}=P_{\eta+1}(z) \exp \left\{\lambda_{\eta+1} z\right\}+\sum_{m=\eta+2}^{q} P_{m}(z) \exp \left\{\lambda_{m} z\right\}
$$

apply the differential operator $D_{\eta+1}^{*}$ which consists in first multiplying both sides by $\exp \left\{-\lambda_{\eta+1} z\right\}$ and then differentiating $(N+1)$ times, in other words

$$
\begin{aligned}
& D^{N+1}\left(\exp \left\{-\lambda_{\eta+1} z\right\} \psi(z) z^{m}\right) \\
& \quad=D^{N+1} P_{\eta+1}(z)+\sum_{m=\eta+2}^{q} D^{N+1}\left(P_{m}(z) \exp \left\{\left(\lambda_{m}-\lambda_{\eta+1}\right) z\right) .\right.
\end{aligned}
$$

The application of this operator has two consequences: first on the left-hand side of (18) it lowers the multiplicity of the zero at $z=0$ by $N+1$ and, secondly, on the right-hand side it destroys the presence of one polynomial coefficient. Thus we are left with an identity of the type

$$
\psi^{*}(z) z^{M-(N+1)}=\sum_{m=\eta+2}^{q} P_{m}^{*}(z) \exp \left\{\lambda_{m}^{*} z\right\},
$$

where the $\lambda_{m}^{*}$ are all distinct, the $P_{m}^{*}(z)$ are all nonzero by assumption and where $\psi^{*}(z) z^{M-(N+1)}$ is an analytic function which by construction has a power series expansion which begins with a power of $z$ greater than or equal to $M-(N+1)$. If we iterate the differential operator $D_{\eta+1}^{*},(q-\eta)$ times, we obtain on the right-hand side a function identically zero and on the left-hand side 
we get an analytic function whose power series begins with a power of $z$ which is greater than or equal to $M-(q-\eta)(N+1)$. If we now recall that $M=N(q-1)$ $+2 q$ we get

$$
M-(q-n)(N+1)=N(q-1)+2 q-(q-\eta)(N+1)=q+(\eta-1) N+\eta,
$$

which is a contradiction if $\eta \geq 1$. Therefore none of the polynomials $P_{m}(z)$ vanishes identically. The validity of the above argument clearly depends on the fact that $f(z)$ itself is not identically zero. But this is clear because by the Lemma at least one polynomial $P_{m}(z)$ is not identically zero. Now we claim that none of the polynomials $U_{m, s}(s)$ in

$$
L_{s}(z)=\sum_{m=1}^{q} U_{s, m}(z) \exp \left\{\lambda_{m} z\right\} \quad(0 \leq s \leq N-1)
$$

can vanish identically. This follows readily from the nonvanishing of the polynomials $P_{m}(z)$ and the fact that if $P(z)$ is a polynomial of degree $N$, then the polynomial $P^{*}(z)$ in

$$
\frac{d}{d z}(P(z) \exp \{\lambda z\})=P^{*}(z) \exp \{\lambda z\}, \quad \lambda \neq 0,
$$

is also of degree $N$.

Step Four. We now ascertain that the $q$ forms $L_{0}(z), \ldots, L_{q-1}(z)$ are linearly independent except for a finite number of values of $z$. In other words, the $q$ by $q$ matrix of coefficients $\left(U_{s, m}(z)\right)$ is not identically zero. But this is clear if we observe that the polynomials $U_{m, s}(z)$ can be defined by

$$
U_{s, m}(z)=\left(\lambda_{m}+D\right)^{s} P_{m}(z), \quad D=\frac{d}{d z},
$$

which is an easy consequence of the formal identity

$$
D^{s}\left(P_{m}(z) \exp \left\{\lambda_{m} z\right\}\right)=\exp \left\{\lambda_{m} z\right\}\left(\lambda_{m}+D\right)^{s} P_{m}(z)
$$

Now, the determinant $\Delta(z)$ of the matrix

$$
\left(U_{s, m}(z)\right)=\left(\begin{array}{ccc}
P_{1}(z) & \cdots & P_{q}(z) \\
\left(\lambda_{1}+D\right) P_{1}(z) & & \left(\lambda_{q}+D\right) P_{q}(z) \\
\cdot & & \cdot \\
\cdot & & \cdot \\
\left(\lambda_{1}+D\right)^{q-1} P_{1}(z) & \cdots & \left(\lambda_{q}+D\right)^{q-1} P_{q}(z)
\end{array}\right)
$$

is not identically zero because the leading coefficient in the expansion of $\Delta(z)$ in powers of $z$ is the product of the leading coefficients of the polynomials $P_{1}(z), \ldots, P_{q}(z)$ and the Vandermonde determinant 


$$
\left(\begin{array}{cccc}
1 & 1 & \cdots & 1 \\
\lambda_{1} & \lambda_{2} & & \lambda_{q} \\
\cdot & \cdot & & \cdot \\
\cdot & \cdot & & \cdot \\
\lambda_{1}^{q-1} & \lambda_{2}^{q-1} & \cdots & \lambda_{q}^{q-1}
\end{array}\right)
$$

which is certainly not zero. Further details can be found in Lang [4, p. 79].

From (19), or otherwise, it readily follows that the degree of $\Delta(z)$ as a polynomial in $z$ is bounded by $N q$. We now want to find a lower bound for the order of the zero of $\Delta(z)$ at $z=0$. Let us therefore multiply the $j$ th column of the matrix (19) by $\exp \left\{\lambda_{j} z\right\}$ and add the new column to the first column for $j=1,2, \ldots, q$. The resulting matrix is

$$
\left(\begin{array}{cccc}
L_{0}(z) & U_{0,2}(z) & \cdots & U_{0, q}(z) \\
L_{1}(z) & U_{2,1}(z) & \cdots & U_{1, q}(z) \\
\cdot & \cdot & & \\
\cdot & \cdot & & \\
L_{q-1}(z) & U_{q-1,2}(z) & \cdots & U_{q-1, q}(z)
\end{array}\right),
$$

whose determinant is still $\Delta(z)$. We now use the fact that

$$
L_{q-1}(z)=z^{M-(q-1)} \psi^{*}(z)=z^{N(q-1)+q+1} \psi^{*}(z),
$$

to obtain $\Delta(z)=z^{N(q-1)+q+1} g(z)$. That is to say, $\Delta(z)$ has a zero at $z=0$ of multiplicity at least $N(q-1)+q+1$. Now, since $\Delta(z)$ is itself a polynomial of degree at most $N q$, it follows that $\Delta(z)$ cannot have a zero of order greater than $N-q-1$ at any other point $z \neq 0$.

Step Five. We now claim that from among the forms $L_{s}(z)(0 \leq s \leq N-1)$, a set of $q$ forms $L_{s_{1}}(z), \ldots, L_{s_{q}}(z)$ can be selected such that the corresponding matrix of coefficients

$$
\left(\begin{array}{ccc}
U_{s_{1}, 1}(z) & \cdots & U_{s_{1}, q}(z) \\
\cdot & & \cdot \\
\cdot & & \cdot \\
U_{s_{q}, 1}(z) & \cdots & U_{s_{q}, q}(z)
\end{array}\right)
$$

is nonsingular at $z=\beta$. In fact we have already seen that if $\beta \neq 0$ and $\Delta(z)$ is 
the determinant of the matrix in (20) we have the factorization $\Delta(z)=$ $(z-\beta)^{e} \psi_{0}(z)$, where $e_{\beta} \leq N-q-1$ and $\psi_{0}(\beta) \neq 0$. Thus solving for $\exp \left\{\lambda_{m} z\right\}$ in the system

$$
L_{s}(z)=\sum_{m=1}^{q} U_{s, m}(z) \exp \left\{\lambda_{m} z\right\} \quad(0 \leq s \leq q-1),
$$

we get the identity

$$
\Delta(z) \exp \left\{\lambda_{m} z\right\}=\sum_{s=0}^{q-1} \Delta_{m, s}(z) L_{s}(z) \quad(1 \leq m \leq q) .
$$

If we differentiate both sides of (21) $e_{\beta}$ times and then substitute the value $z=\beta$ we get the linear system

$$
\begin{aligned}
\Delta^{(e} \beta^{\prime}(\beta) \exp \left\{\lambda_{m} \beta\right\} & =\sum_{s=0}^{q+e} \beta_{m, s}^{-1} \Delta_{s}^{*}(\beta) \\
& =\sum_{n=1}^{q}\left(\sum_{s=0}^{q+e} \beta^{-1} \Delta_{m, s}^{*} U_{s, n}(\beta)\right) \exp \left\{\lambda_{n} \beta\right\} \quad(1 \leq m \leq q),
\end{aligned}
$$

from which we get the identity

$$
\Delta^{\left(e_{\beta}\right)}(\beta) I_{q \times q}=\Delta^{*} \cdot U,
$$

where $I_{q \times q}$ is the identity matrix of order $q, \Delta^{*}$ is the matrix of coefficients

$$
\Delta^{*}=\left(\Delta_{m, s}^{*}\right) \quad\left(1 \leq m \leq q ; 0 \leq s \leq q+e_{\beta}-1\right),
$$

and $U$ is the matrix

$$
U=\left(U_{s, n}(\beta)\right) \quad\left(0 \leq s \leq q+e_{\beta}-1 ; 1 \leq n \leq q\right) .
$$

Now, since $\Delta^{(e} \beta^{\prime}(\beta) \neq 0$, we know from (22) that the rank of the matrix $\Delta U$ is $q$ and hence the rank of each factor must also be $q$. Using the inequality $e_{\beta} \leq N-q-1$ we see that the above statement is equivalent to the assertion that from among the $N-1$ forms $L_{0}(\beta), \ldots, L_{N-2}(\beta)$ we can select $q$ forms which are linearly independent. That is to say, there are $q$ forms whose corresponding matrix of coefficients is nonsingular at $z=\beta$. Without loss of generality we may assume that

$$
\hat{L}_{1}(\beta), \hat{L}_{2}(\beta), \ldots, \hat{L}_{q}(\beta)
$$

are $q$ chosen linearly independent forms.

To get an estimate of the size of the forms $L_{s}(\beta)$ we simply observe that by the Cauchy integral formula for the derivative of a function we have

$$
L_{s}(\beta)=f^{(s)}(\beta)=\frac{s !}{2 \pi i} \int_{|z-\beta|=1} \frac{f(z) d z}{(z-\beta)^{s+1}} .
$$


Taking absolute values we obtain

$$
\left|L_{s}(\beta)\right| \leq N ! \underset{|z-\beta|=1}{\operatorname{Max}}|f(z)| \quad(0 \leq s \leq N-1) .
$$

A bound for $\operatorname{Max}|z-\beta|=1|f(z)|$ follows readily from (11) and (14)

$$
\begin{aligned}
\underset{|z-\beta|=1}{\operatorname{Max}}|f(z)| & \leq(1+|\beta|)^{M} \underset{|z-\beta|=1}{\operatorname{Max}}|\psi(z)| \\
& \leq H^{q N} \exp \left\{-(q N / 2) \log N+c_{12} p H+4 N q^{2} \log p\right\},
\end{aligned}
$$

where we have used the fundamental inequality

$$
|\beta| \leq \text { (height of } \beta) \times(\text { degree of } \beta) \leq H d \text {. }
$$

Hence we get

$$
\begin{array}{r}
\left|L_{s}(\beta)\right| \leq H^{q N} \exp \left\{-(q N / 3) \log N+c_{12} p H+4 N q^{2} \log p\right\} \\
(0 \leq s \leq N-1) .
\end{array}
$$

In particular the bound in (25) applies to all the forms in (23). The inequality in (25) holds on the assumption that $p \geq c_{13}$. The constant $c_{12}=2 d c_{8}$.

We now consider the system in (5) of $\tau$ linearly independent forms

$$
\phi_{j}(\beta)=\sum_{m=1}^{q} A_{i, m}(\beta) \exp \left\{\lambda_{m} \beta\right\}, \quad(1 \leq j \leq \tau)
$$

and complete the system by choosing $q-\tau$ forms from among those in (23), say $\hat{L}_{1}(\beta), \ldots, \hat{L}_{q-\tau}(\beta)$ such that the resulting system

$$
\phi_{1}(\beta), \ldots, \phi_{\tau}(\beta), \hat{L}_{1}(\beta), \ldots, \hat{L}_{q-\tau}(\beta)
$$

has a nonsingular matrix of coefficients

$$
Q=\left(\begin{array}{ccc}
A_{1,1}(\beta) & \cdots & A_{1, q}(\beta) \\
\cdot & & \cdot \\
\cdot & & \cdot \\
A_{\tau, 1}(\beta) & \cdots & A_{\tau, q}(\beta) \\
\hat{U}_{1,1}(\beta) & \cdots & \hat{U}_{1, q}(\beta) \\
\cdot & & \cdot \\
\cdot & & \cdot \\
\hat{U}_{q-\tau, 1}(\beta) & \cdots & \hat{U}_{q-\tau, q}(\beta)
\end{array}\right)
$$

Step Six (Final balancing act). To complete the proof of Theorem 1 we now 
find upper and lower bounds for the determinant of the matrix $Q$ in (26a). To obtain an upper bound we use the estimates (25) and the inequalities for $\phi_{1}(\beta), \ldots$, $\phi_{\tau}(\beta)$ in (5). To obtain a lower bound we use the simple fact that the determinant of $Q$ is a nonzero algebraic number and hence, if multiplied by a suitable denominator, the resulting number will be an algebraic integer whose norm has absolute value greater than one.

For each $m, 1 \leq m \leq q$, we now multiply the $m$ th column in (26a) by $\exp \left\{\lambda_{m} \beta\right\}$ and add the resulting column to the first column to obtain the matrix

$$
Q^{*}=\left(\begin{array}{cccc}
\phi_{1}(\beta) & A_{1,2}(\beta) & \cdots & A_{1, q}(\beta) \\
\cdot & \cdot & & \cdot \\
\phi_{\tau}(\beta) & A_{\tau, 2}(\beta) & \cdots & A_{\tau, q}(\beta) \\
\hat{L}_{1}(\beta) & \hat{U}_{1,2}(\beta) & \cdots & \hat{U}_{1, q}(\beta) \\
\cdot & \cdot & & \cdot \\
\cdot & \cdot & & \cdot \\
\hat{L}_{q-\tau}(\beta) & \hat{U}_{q-\tau, 2}(\beta) & \cdots & \hat{U}_{q-\tau, q}(\beta)
\end{array}\right)
$$

To estimate the determinant of the matrix in (26b) we use the fundamental inequality (24) to obtain

$$
\overline{\left|A_{i, m}(\beta)\right|} \leq d A(d H)^{d} .
$$

From (15), (17) and (24) we obtain

$$
\overline{\left|U_{s, m}(\beta)\right|} \leq H^{N} \exp \left\{3 N \log N+4 N q^{2} \log p\right\}
$$

provided $p \geq c_{14}$

The estimates (27) and (28) imply that the determinant of the matrix in (26b) is bounded by

$$
|\operatorname{det} Q| \leq\left(\left|\phi_{*}(\beta)\right|+L_{*}(\beta) \mid\right) H^{d \tau+N(q-\tau)} \exp \left\{(q-\tau)\left(4 N \log N+4 N q^{2} \log p\right)\right\}
$$

provided that $p \geq c_{15}$. In (29) we have put $\left|\phi_{*}(\beta)\right|=\operatorname{Max}_{1 \leq j \leq i}\left|\phi_{j}(\beta)\right|$ and $\left|L_{*}(\beta)\right|$ $=\operatorname{Max}_{1 \leq j \leq q-r}\left|\hat{L}_{j}(\beta)\right|$.

To obtain a lower bound for $\operatorname{det} Q$, we observe that $\operatorname{det} Q$ is an algebraic number in the field $K(\beta)$ which is of degree at most $\nu d$ over the rationals. Therefore, if the leading coefficient of the irreducible equation defining $\beta$ is $T=T(\beta)$ $\leq H$, we have that 


$$
Q_{0}=T^{d(d \tau+N(q-\tau))} \operatorname{det} Q
$$

is a nonzero algebraic integer in the field $K(\beta)$, and its norm has absolute value greater than one. Now, using (26a), (27) and (28) we get the following bound for each of the conjugates of the number $Q_{0}$

$$
\overline{\left|Q_{0}\right|} \leq H^{(d+1)(d \tau+N(q-\tau))} \exp \left\{(q-\tau)\left(4 N \log N+4 N q^{2} \log p\right)\right\},
$$

provided $p \geq c_{15}$.

Finally we get

$$
\begin{aligned}
& 1 \leq \prod_{i} i\left(Q_{0}\right) \leq\left|Q_{0}\right| H^{(d \nu-1)(d+1)(d r+N(q-\tau))} \\
& \cdot \exp \left\{(d \nu-1)(q-\tau)\left(4 N \log N+4 N q^{2} \log p\right)\right\},
\end{aligned}
$$

where the symbol ' $i$ ( )' means the $i$ th conjugate of a number in the field $K(\beta)$; the last inequality we can also write as

(31) $|\operatorname{det} Q| \geq H^{-2 \nu d^{2}(d \tau+N(q-\tau))} \exp \left\{-(d \nu-1)(q-\tau)\left(4 N \log N+4 N q^{2} \log p\right)\right\}$.

The inequalities (29) and (31) now give

$$
1 \leq\left(\left|\phi_{*}(\beta)\right|+\left|L_{*}(\phi)\right|\right) H^{3 \nu d^{2}(d r+N(q-\tau))}
$$

provided $p \geq c_{15}$.

$$
\cdot \exp \left\{d \nu(q-\tau)\left(4 N \log N+4 N q^{2} \log p\right)\right\}
$$

If we now put $N=H^{6}$ and observe that $q-r \leq \nu \sigma q^{(\nu-1) / \nu}$ and $p<q^{1 / \nu}$ we get

$$
\left|L^{*}(\beta)\right| \leq \exp \left\{-(q N / 6) \log N+4 N q^{2} \log p\right\} .
$$

We can also write (32) in the form

$$
\begin{gathered}
1 \leq\left(\exp \left\{-\Lambda H^{7}\right\}+\exp \left\{-(q N / 6) \log N+4 N q^{2} \log p\right\}\right) \\
\cdot\left(\exp \left\{c_{16} q^{(\nu-1) / \nu}\left(4 N \log N+4 N q^{2} \log q\right)\right\}\right) .
\end{gathered}
$$

We now choose $q$ as a function $N$ so that $c_{16} q^{(\nu-1) / \nu} 4 N q^{2} \log q \leq(q N \log N) / 24$, i.e., we take $q=c_{18}((\log N) / \log \log N)^{\nu /(2 \nu-1)}$, with a suitable constant $c_{18}$.

Clearly, $4 N q^{2} \log p \leq(1 / 24) q N \log N$ and $4 c_{16} q^{(\nu-1) / 2} N \log N \leq(1 / 24) q N \log N$ if $q \geq c_{19^{\circ}}$ Hence we can write the inequality (33) as

$$
\left.1 \leq\left(\exp \left\{-\Lambda H^{7}\right\}+\exp \left\{-(3 / 24)_{q} N \log N\right\}\right)\left(\exp \{1 / 12)_{q N} \log N\right\}\right)
$$

which is a contradiction if $H \geq c_{20}$, or equivalently if $\Lambda \geq c_{21}$. This then implies that $|\phi(\beta)| \geq \exp \left\{-\Lambda H^{7}\right\}$. This completes the proof of Theorem 1 . 
The author wishes to express his gratitude to the referee without whose many suggestions the paper would have contained many obscurities.

\section{BIBLIOGRAPHY}

1. N. I. Fel'dman, Arithmetic properties of the solutions of a transcendental equation, Vestnik Moskov. Univ. Ser. I Mat. Meh. 1964, no. 1, 13-20; English transl., Amer. Math. Soc. Transl. (2) 66 (1968), 145-153. MR 28 \# 2091.

2. A. Gel'fond, Transcendental and algebraic numbers, GITTL, Moscow, 1952; English transl., Dover, New York, 1960. MR 15, 292; 22 \# 2598.

3. C. Hermite, Sur la fonction exponentielle, Acad. Sci. Paris 77 (1873); Ouvres. III, 150-181.

4. S. Lang, Introduction to transcendental numbers, Addison-Wesley, Reading, Mass., 1966. MR 35 \# 5397.

5. F. Lindemann, Über die Zahl $\pi$, Math. Ann. 20 (1882).

6. K. Mahler, Zur Approximation der Exponential Funktion und des Logarithmus, J. Reine Angew. Math. 166 (1931/32).

7. D. Mardohai-Boltouski, On some properties of transcendental numbers of the first class, Mat. Sb. 34 (1927).

8. C. J. Moreno, The values of exponential polynomials at algebraic points. II (to appear).

9. A. Sidlovskiī, On the transcendence of the values of a class of entire functions satisfying linear differential equations, Dokl. Akad. Nauk SSSR 105 (1955), 35-37. (Russian) MR 17, 947.

10. C. Siegel, Über einige Anwendungen Diophantischer Approximationen, Abh. Preuss. Akad. Wiss. 1929/30, No. 1.

11. - Transcendental numbers, Ann. of Math. Studies, no. 16, Princeton Univ. Press, Princeton, N.J., 1949. MR 11, 330.

THE CENTER FOR ADVANCED STUDY, UNIVERSITY OF' ILLINOIS, URBANA, ILLINOIS 61801

DEPARTMENT OF MATHEMATICS, UNIVERSITY OF ILLINOIS AT URBANA, URBANA, ILLINOIS 61801 (Current address) 